\title{
Renewable energy-based plant remote monitoring complex using Wi-Fi channels and elements of artificial vision
}

\author{
S. E. Shcheklein, Yu. E. Nemikhin, S. V. Nevyantsev, \\ S. A. Korzhavin, A. O. Postovalov, D. A. Nosov \\ \& Yu. Z. Zagafuranova \\ Department of Nuclear Power Plants and Renewables, \\ Ural Federal University, Russia
}

\begin{abstract}
This paper presents an analysis of the renewable energy-based plant operation using special systems designed for monitoring the plant operation parameters and for performing the atmospheric parameters control.

Keywords: renewable energy, monitoring, artificial vision.
\end{abstract}

\section{Introduction}

Combining traditional power sources with renewable energy sources is one of the most promising directions for the development of small-scale power generation. Renewable energy sources offer a number of advantages:

- increased efficiency of small-scale power generation resulted from energy savings (substitution of fuel and electric energy);

- improved reliability of electric power supply from neighboring renewable sources to local energy consumers; and

- minimized negative impact on the environment resulted from reduced atmospheric pollution.

Along with the advantages, however, there are also some disadvantages of using renewable energy sources, which include their non-uniform operating availability in regions and times, and high capital costs per unit of the installed capacity. These factors complicate the problems to be solved in selecting the structure and setting the parameters of power generation systems, which are 
determined not only by the interdependent processes of generating and consuming energy but also by the reliability requirements to their stable functioning.

On the one hand, renewable energy-based stand-alone installations allow them to be regarded as local power systems supplying energy to remote consumers. On the other hand, renewable energy is delivered to the local power system from the surrounding environment. Therefore, this object of our research should be regarded as an element of a more complicated system. System approach-based methods are applicable to solving such problems; and making a decision with regard to the optimal scheme for the power supply should be based on effectiveness criteria considering the environmental implications. In evaluating the influence of external factors, the major problem is to obtain statistically significant evaluation results in view of their uneven timing. One of the ways to solve this problem is to describe the external factors with allowance made for their nonsteady statistical behaviour [1].

\section{Measurement data acquisition system}

A measurement data acquisition system was specifically designed to obtain synchronous statistical data on various forms of renewable energy flows (solar, wind, ambient air and soil temperatures) from the environment and to ensure effective use of the energy flows by advanced technologies. The measurement data acquisition system was implemented in a set of installations, which characteristic input data are reviewed in Table 1.

The measurement data acquisition system is based on a NI CompactRIO (Compact Reconfigurable Input Output) programmable platform, which is an embedded multifunctional platform for data acquisition and parameter control, designed for performing tasks that call for high productivity and reliability standards [2].

The NI CompactRIO is an embedded instrumentation management system, which is based on the technology of configurable input/output, NI RIO. The system comprises chassis equipped with a programmable logic device (PLD), a real-time controller, and input/output modules (fig. 1).

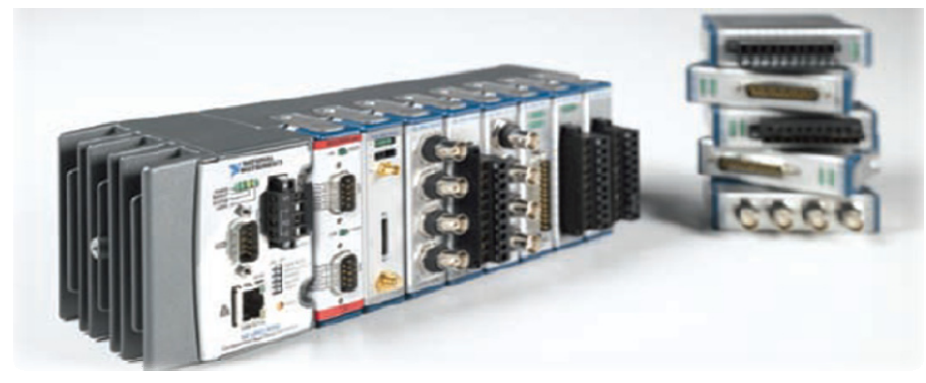

Figure 1: $\quad$ NI CompactRIO platform. 
Table 1: $\quad$ Characteristic input data.

\begin{tabular}{|l|l|}
\hline Installation & Key characteristics reviewed \\
\hline Meteorological complex & - temperature \\
& - humidity \\
& - precipitation \\
& - wind speed \\
& - wind direction \\
& - total solar radiation \\
& - IR solar radiation \\
& - UV solar radiation \\
\hline Photoelectric multiplier & - output voltage \\
& - current \\
& - power \\
\hline Wind power plant & - output voltage \\
& - current \\
& - power \\
& - rotational speed \\
\hline Solar collector & - input temperature \\
& - output temperature \\
& - coolant rate \\
& - heating power \\
\hline Biogas unit & - input temperature \\
& - output temperature \\
& - coolant rate \\
\hline Solar concentrator & - heating power \\
\hline & - input temperature \\
& - output temperature \\
& - coolant rate \\
- heating power \\
\hline & - biomass temperature \\
& - temperature in bioreactor \\
& - biogas flow rate \\
& - biogas pressure \\
& - pH index in bioreactor \\
\hline & \\
\hline
\end{tabular}

Because of widely separated locations of the renewable energy-based plants over a considerable territory, which is not coverable with unified fiber-optic network, the local measurement complexes and the central platform server are networked via Wi-Fi-based channels.

Using the measurement data acquisition system, a continuous collection of data is processed from more than 100 primary transmitters and 3 fast video cameras installed for monitoring the parameters and for processing the images obtained from wind, photoelectric, biogas and other renewable energy-based installations, which are distributed over the Ural Federal University site (fig. 2). The measurement data acquisition system is used for data collection and for 
1188 Energy Production and Management in the 21st Century, Vol. 2

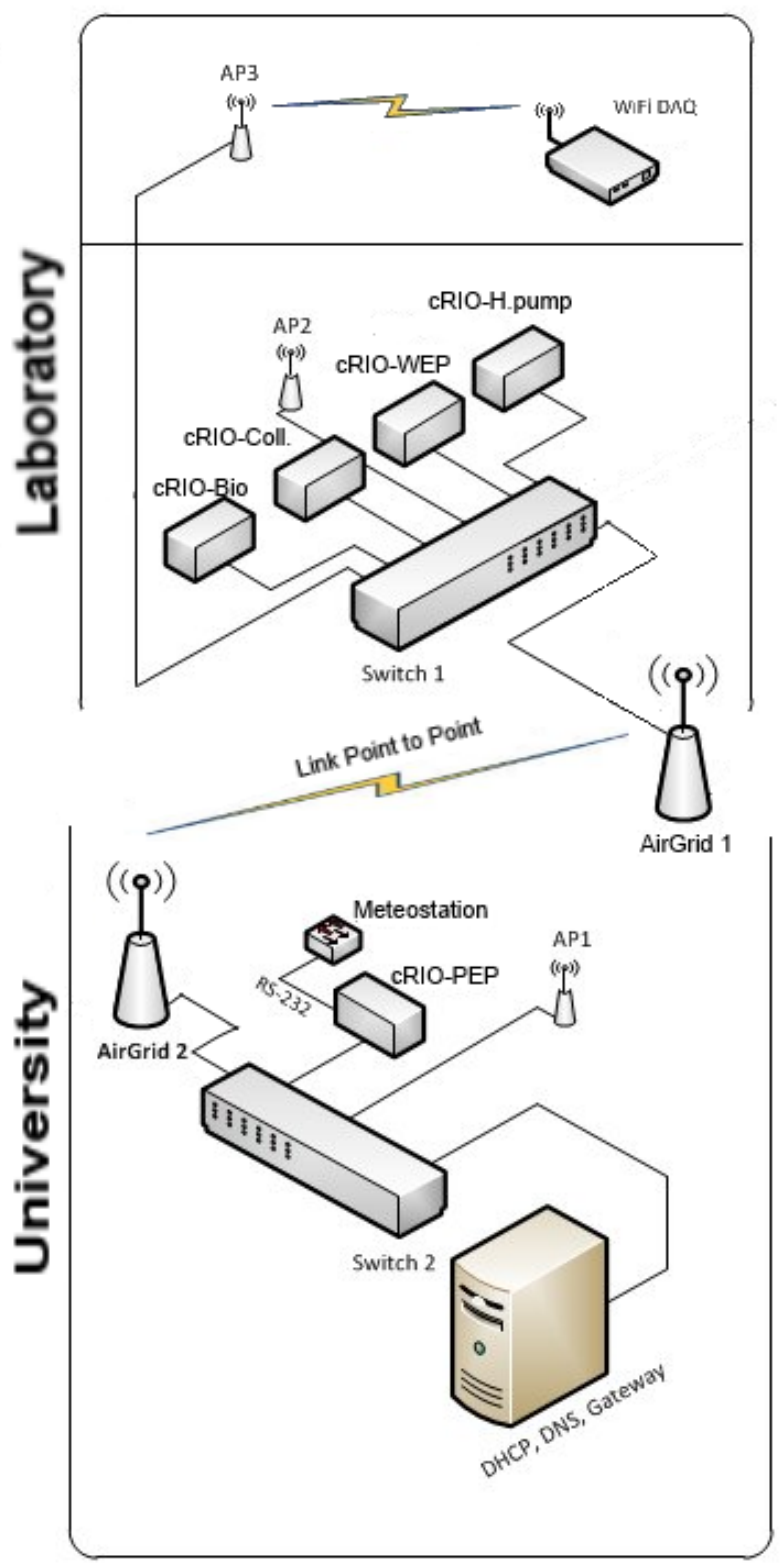

Figure 2: Installation layout on site of the Ural Federal University.

Wi-Fi broadcasting the data to the server and peripheral workstations capable of performing subsequent data analysis and processing.

For on-line monitoring the characteristics of renewable energy-based plants, a software package was designed in the NI LabVIEW environment, with its screen interface presented in fig. 3 . 
When referencing a specific installation, the help interface visualizes certain points and characteristics being measured in the form of a measurement data flowchart (fig. 4).

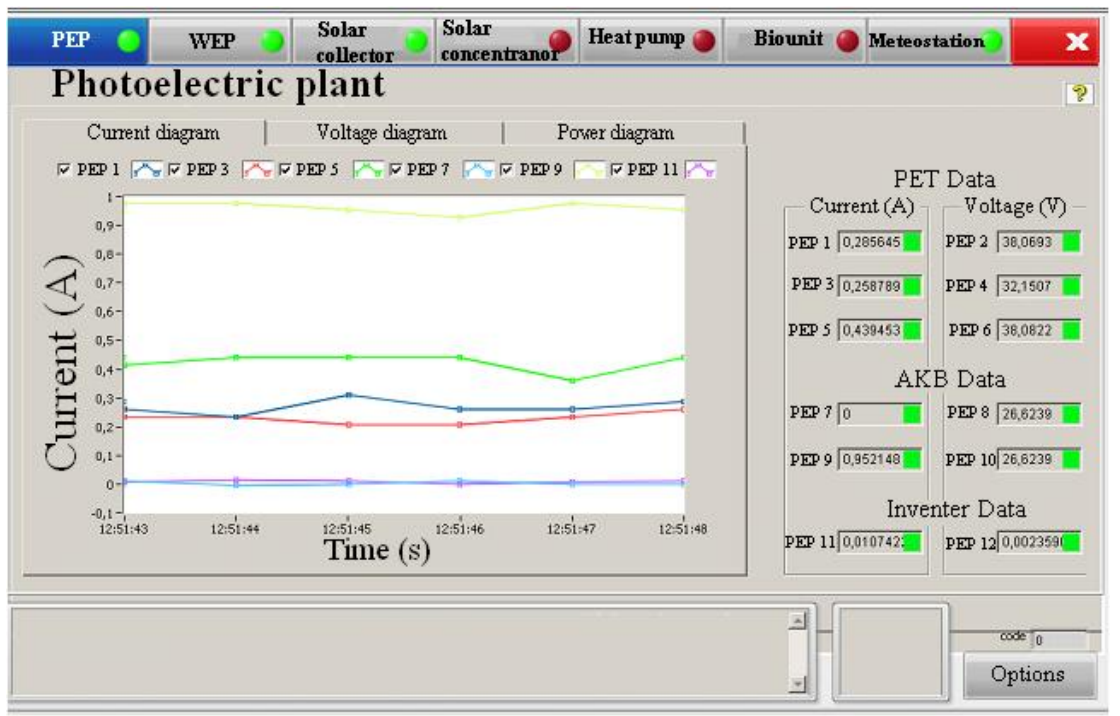

Figure 3: Monitoring system screen interface.

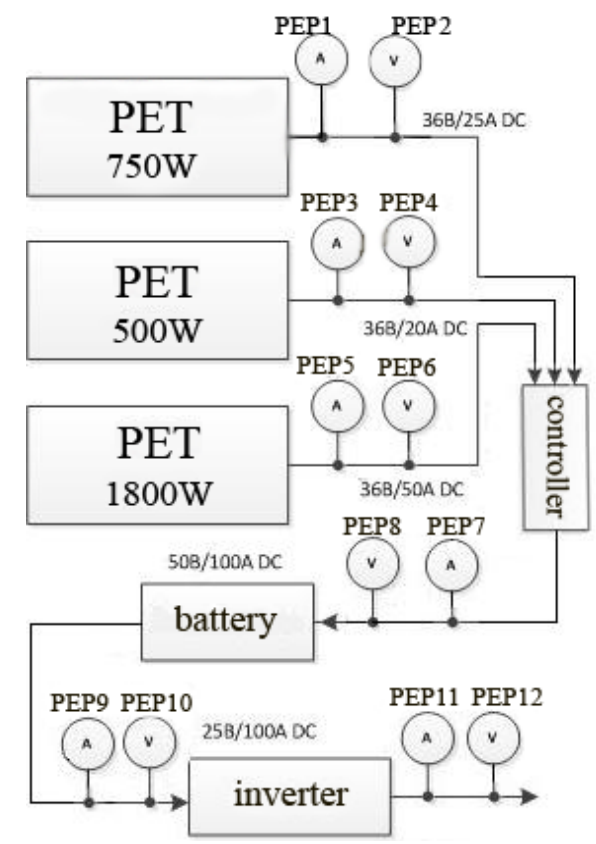

Figure 4: $\quad$ Photoelectric plant measurement point flowchart. 
The designed measurement data acquisition system can be used for creating measurement data arrays grouped by a time interval of one second to one month, for performing statistical data manipulation, for storing primary measurements data, and for processing the results in the server buffer pool memory.

\section{First results}

Figures 5 and 6 display the daily measurement results obtained for the photoelectric and solar collector systems.

The measurements results obtained over one-year and many-year cycles are processed to identify characteristic changes in natural climate patterns and to estimate energy flows in view of the typical frequency and time scale of the processes. Figures 7, 8, and 9 represent the many-year cycle data on the variations in the solar radiation and wind speed observed in the conditions of the Urals region.

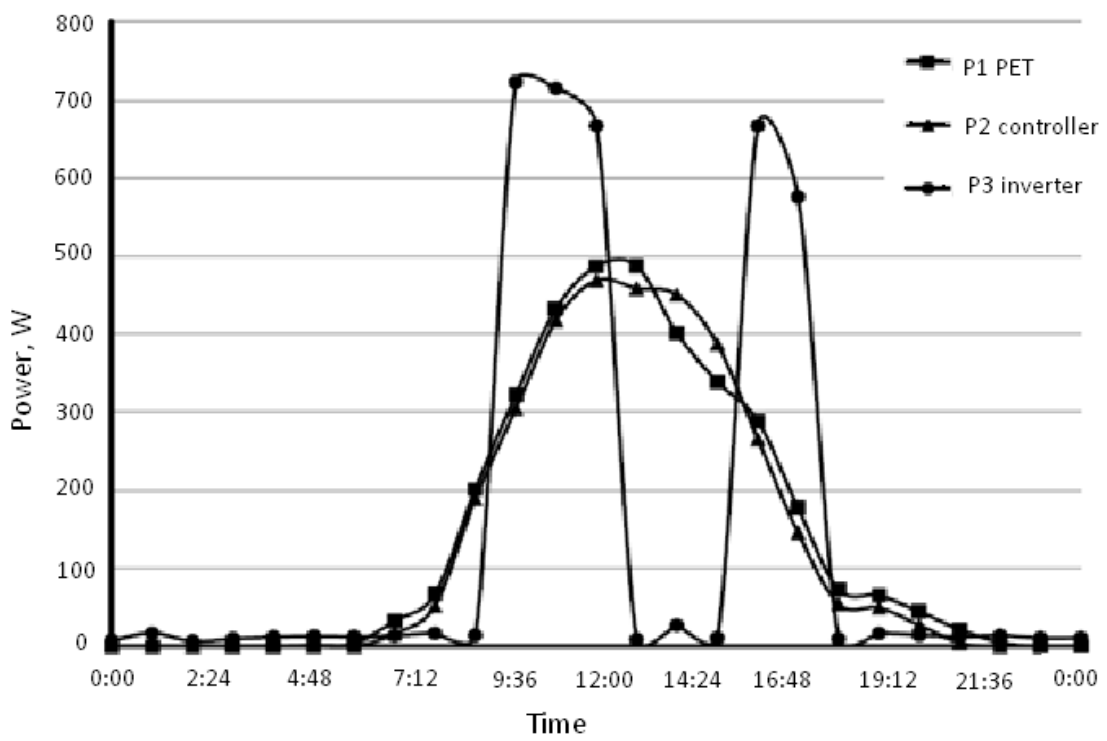

Figure 5: Power variations in the characteristic points of photoelectric plants (at an external load of $300 \mathrm{~W}$ ). 


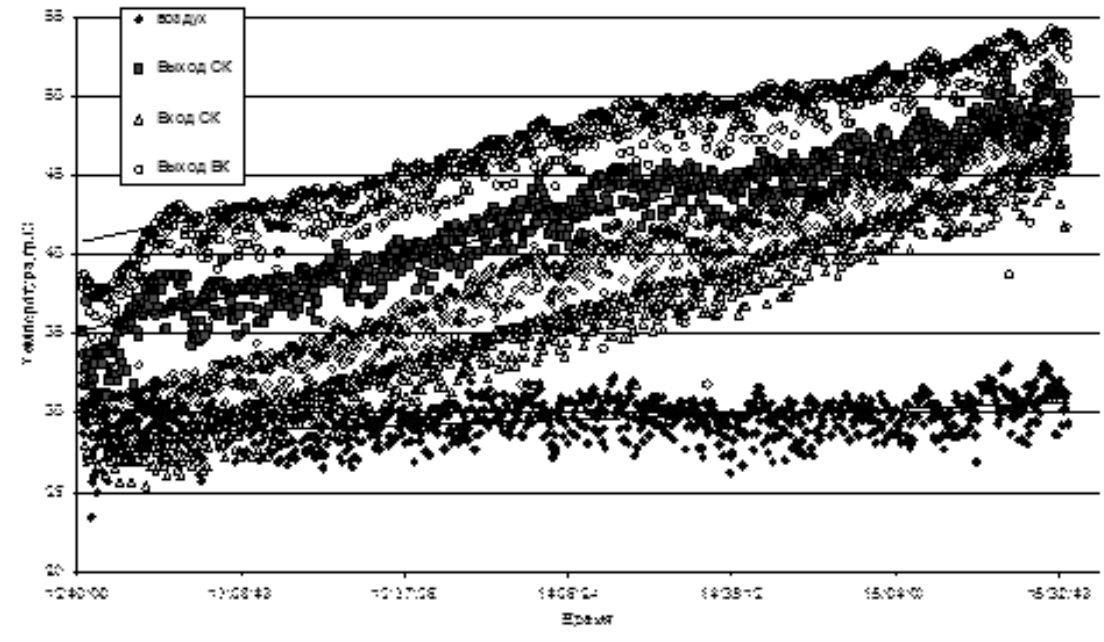

Figure 6: Daily temperature measurements in flat-plate and vacuum solar collectors.

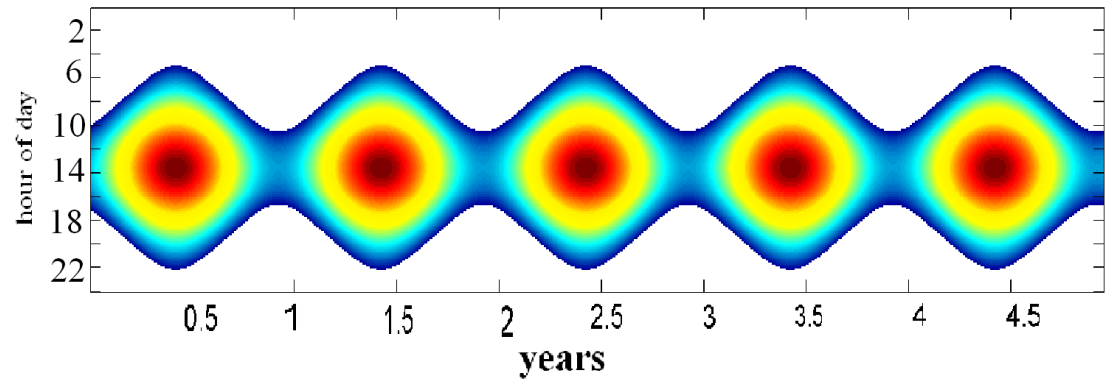

Figure 7: Assumption diagram illustrating variations in the intensity of extraterrestrial solar radiation. 


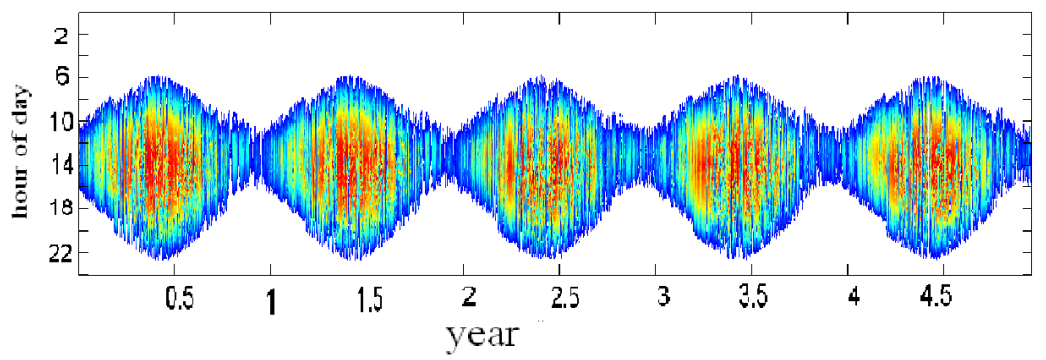

Figure 8: Experimental chart illustrating the measured values of solar radiation intensity towards the horizontal face.

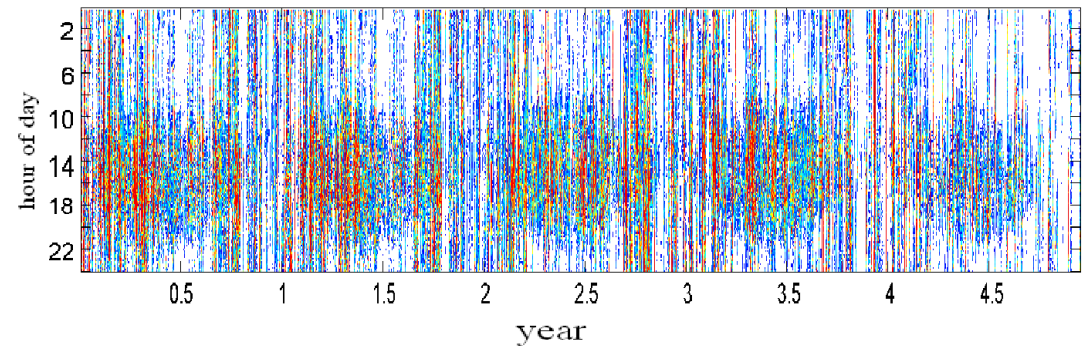

Figure 9: Experimental chart of wind speed variations.

Figures 10-13 represent the process spectra obtained by using the discrete Fourier transform algorithm [3-6]. In the spectra, the characteristic peaks at a certain frequency correspond to every periodic component.
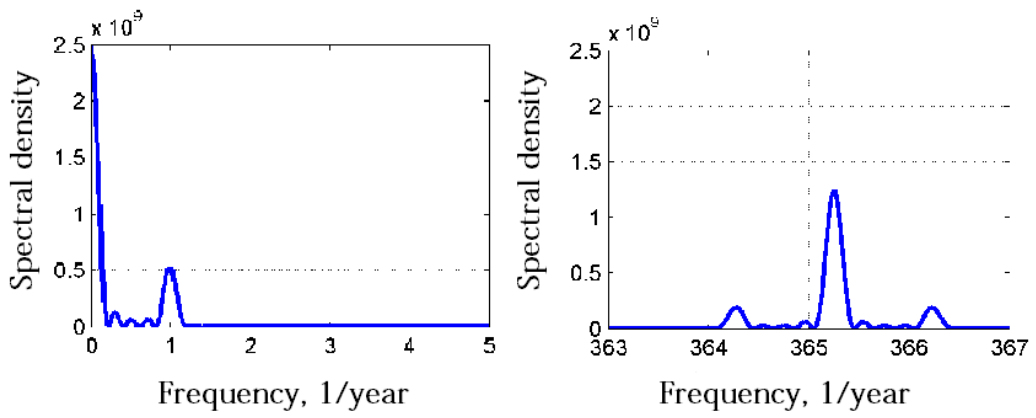

Figure 10: Spectral density of extraterrestrial solar radiation intensity. 

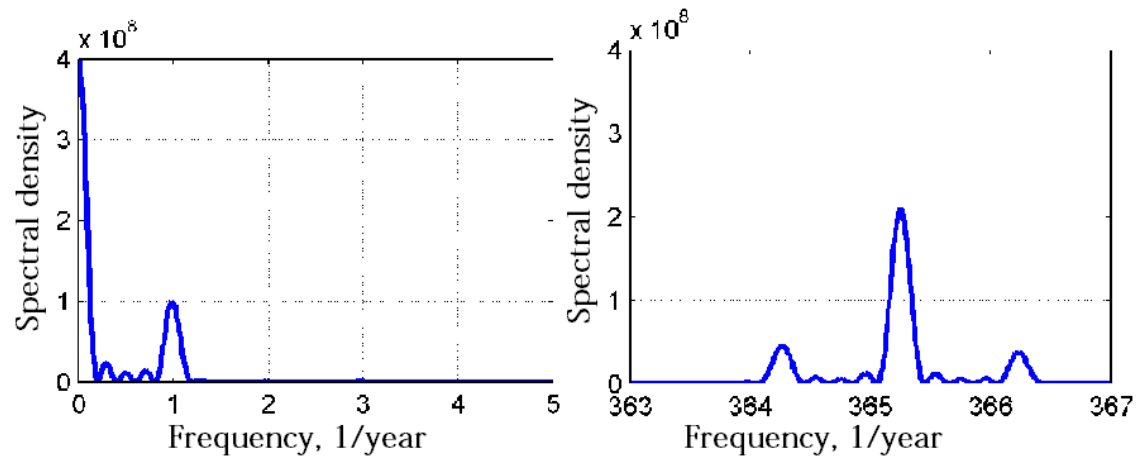

Figure 11: Spectral density of solar radiation intensity.
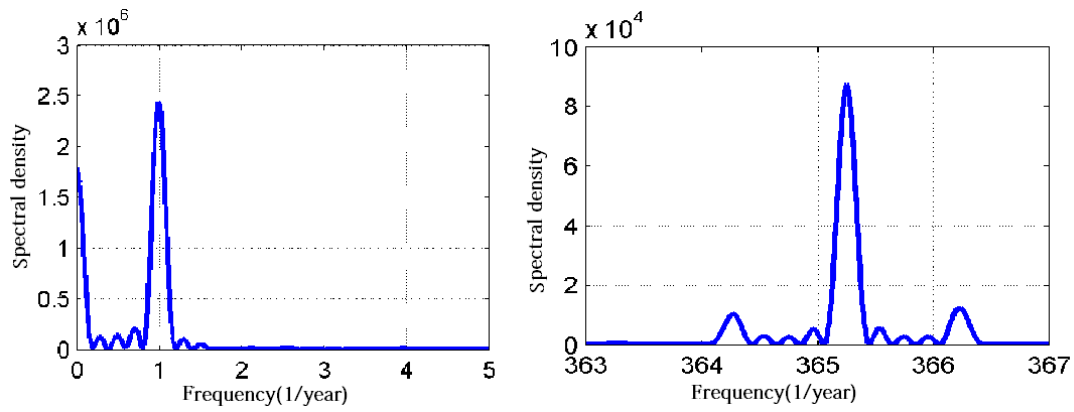

Figure 12: Spectral density of atmospheric temperature.
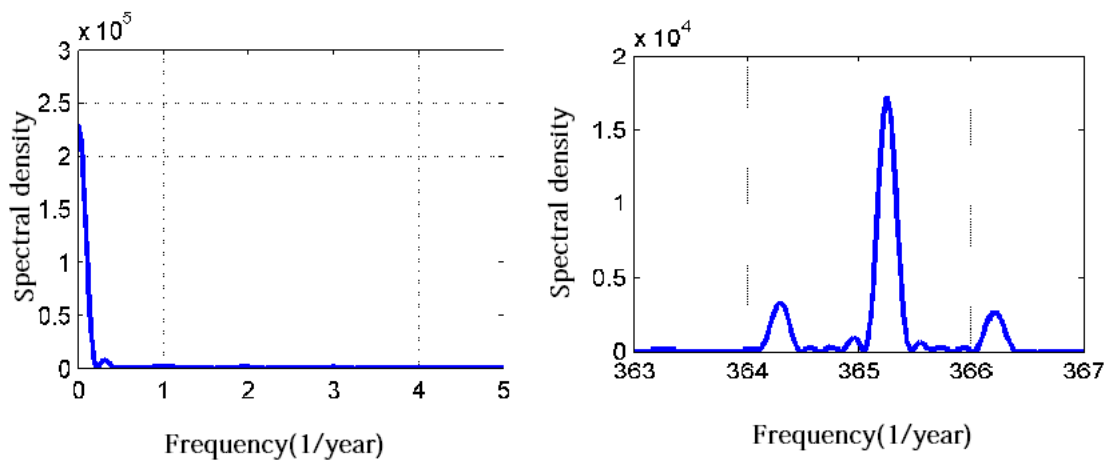

Figure 13: Spectral density of wind speed.

The spectral characteristics demonstrate that the observed climatic processes are characterized with the same frequency behaviour. This means that extraterrestrial solar radiation has a considerable impact on the formation of the 
processes observed. The spectral density maximums correspond to the yearly and daily periodic components. The secondary maximums near the daily components are attributed to the frequency modulation of oscillations effect.

\section{Conclusion}

Thus gathered data were used to design a mathematical model [1] simulating receipts of renewable energy; the mathematical model refines the estimated characteristics of the power plants and provides data of improved accuracy, as compared to the known numerical schemes and computational techniques [7-9] that are not favored by being based on results of long-run and deliberate monitoring specifically performed for certain area.

\section{References}

[1] Shcheklein, S.E. \& Vlasov, V.V., Modelling of nonsteady random processes in the tasks of justification of renewable sources of energy. International scientific journal for alternative energy and ecology, $\mathbf{3}$, pp. 67-71, 2012.

[2] http: ॥www.ni.com - National instruments.

[3] Anderson T., The Statistical Analysis of Time Series. Mir: Moscow, pp. 1$75,1976$.

[4] Bendat, G. \& Pirsol, A., Application Analysis of Random Data, Mir: Moscow, pp. 50-86, 1989.

[5] Bendat, G. \& Pirsol, A., Implementation of Correlational and Spectral Analysis, Mir: Moscow, pp. 237-251, 1983.

[6] Box, G. \& Jenkins, G., Analysis of Time Series. Prognosis and Management, Mir: Moscow, pp. 291-308, 1974.

[7] Vlasov, V., Nemikhin, Yu. \& Shcheklein, S. The use low hydropower possibilities for the increase of the auxiliaries redundancy reliability, Tenth International Conference Advanced Methods in the Theory Of Electrical Engineering, AMTEE 11, Klatovy, pp. 23-24, 2011.

[8] Vasko, P.F., Technical Efficiency Calculation of Implementation of Windpower Plants Based On the Results of Hourly Measurements of the Wind Speed, Technical electrodynamics, 6, pp. 45-49, 2001.

[9] Jangamshetti Suresh H., Optimum siting of wind turbine generators, IEEE Trans. Energy Convers, V. 16, 1, pp. 8-13, 2001.

[10] Weisser, D. \& Foxon, T.J., Implications of seasonal and diumal variations of wind velocity for power output estimation of a turbine: a case study of Grenada, Int. J. Energy Res., V. 27, 13, pp. 10-17, 2003. 